\title{
Design and Optimization of Large-section Profile Die for AZ80 Alloy
}

\author{
Minglong MA $^{a^{*}}$, Haizhen WANG, Kui ZHANG ${ }^{b}$, Jiawei YUAN, Ting LI \\ State Key Laboratory of Non-ferrous Metals and Processes \\ General Research Institute for Non-ferrous Metals, Beijing, 100088 \\ amaminglong@grinm.com, b'zhkui@grinm.com
}

\begin{abstract}
Keywords: AZ80 Alloy, Large-section Profile, Die Optimization
Abstract. This paper regards a kind of AZ80 magnesium alloy extrusion die as the research object to design its shape and make the systematic optimization. The results show that after optimization, the simulated extruding warpage reduced with stable dimension. By comparing the loads of dummy blocks under different die designs, the loads are close when the profile is extruded to the stable stage. After adding the diversion trench, the alloy is easier to become the desired shape through the secondary deformation.
\end{abstract}

\section{Introduction}

Compared with that of aluminum, copper and other metal alloys, the plastic processing technology of magnesium alloy is relatively backward. This is because that magnesium alloy has poor plasticity and is mostly shaped by extruding at high temperature [1]. During extrusion deformation, the deformation body is subjected to three-dimensional compressive stress in the extrusion container, which is conductive to giving full play to the plasticity. However, the extrusion technique of magnesium alloy is very complex and both the extrusion deformation performance and the structure property of the extruding products are affected by the structural state of the extrusion billet, extrusion temperature and speed and lubrication condition [2]. In particular, the techniques of the wide, flat and thin-walled hollow profile with complex cross section shape and the solid profile with stiffened plate are more difficult with rare research at present [3]. For the parts with greatly different wall thicknesses, especially the thin-walled part far away from the extrusion center, the strong pull force produces great untouched deformation, making the wall thinner. For the profile with thick wall, due to the fast flow in the middle part, it's easy to produce the untouched deformation to make it thinner. For the specially complicated profile, due to the production of severe distortion, it's difficult to straighten it or ensure the precision of dimension tolerance, or the service life of the die is short, leading to the increase of the cost and the difficulty in maintaining production. To solve this problem, this paper regards a kind of magnesium alloy extruding die as the research object for optimization to make the basic preparation for extruding the high-quality magnesium alloy profile. The extrusion temperature and speed commonly selected in the die optimization process are $350^{\circ} \mathrm{C}$ and $1 \mathrm{~mm} / \mathrm{s}$, respectively.

\section{Machining Size Design of Profile Die Holes and Die Hole Arrangement}

For profile, the size of die hole can be generally calculated by the following equation [4]:

$$
A=A_{0}+M+\left(K_{y}+K_{p}+K_{T}\right) A_{0}
$$

where $A_{0}$ is the nominal size of the profile section; $M$ is the permissible variation of nominal size of the profile; $K_{y}$ is the coefficient considering the partial dimension reduction of the profile due to the pull force for the T-section and slotted profiles; $K_{p}$ is the coefficient considering the dimension reduction in stretch flattening; $K_{T}$ is the heat shrinkage of the profile, in which $K_{T}=t \alpha-t_{1} \alpha_{1}, t$ and $t_{1}$ are the heating temperatures of billet and die, respectively; $\alpha$ and $\alpha_{1}$ are the linear expansion coefficients of the extruded profile and the die material under the extrusion temperature, respectively.

The profile material is AZ80 magnesium alloy and the die material is $3 \mathrm{Cr} 2 \mathrm{~W} 8 \mathrm{~V}$. The coefficient $K_{T}$ is determined first. For AZ80 magnesium alloy, $\alpha=27.3 \times 10^{-6}, t=350^{\circ} \mathrm{C}$; for $3 \mathrm{Cr} 2 \mathrm{~W} 8 \mathrm{~V}, \alpha=14 \times 10^{-6}$, 
$\mathrm{t}_{1}=360^{\circ} \mathrm{C}$, so $K_{T}=0.004515 . K_{y}$ and $K_{p}$ can be inquired from Table 1 according to the profile cross section dimension.

Table 1 The values of $K_{v}$ and $K_{p}$ in Eq. 1

\begin{tabular}{cccccc}
\hline $\begin{array}{c}\text { size of the profile } \\
\text { section } / \mathrm{mm}\end{array}$ & $K_{y}$ & $K_{p}$ & $\begin{array}{c}\text { size of the profile } \\
\text { section } / \mathrm{mm}\end{array}$ & $K_{y}$ & $K_{p}$ \\
\hline $1-3$ & $0.04-0.03$ & $0.03-0.02$ & $61-80$ & $0.004-0.005$ & $0.006-0.007$ \\
$4-20$ & $0.02-0.01$ & $0.02-0.01$ & $81-120$ & $0.003-0.004$ & $0.005-0.006$ \\
$21-40$ & $0.007-0.006$ & $0.007-0.008$ & $121-200$ & $0.002-0.003$ & $0.005-0.0045$ \\
$41-60$ & $0.005-0.006$ & $0.0065-0.0075$ & $>200$ & $0.001-0.0015$ & $0.002-0.003$ \\
\hline
\end{tabular}

After calculation, the large-section profile dimension and the die orifice dimension are shown in Fig.1.

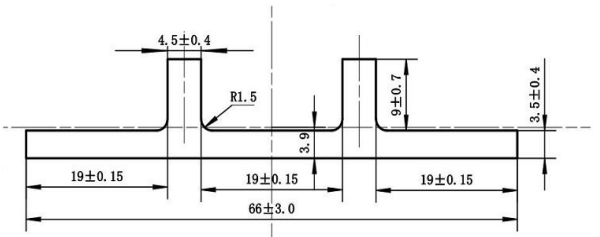

(a)

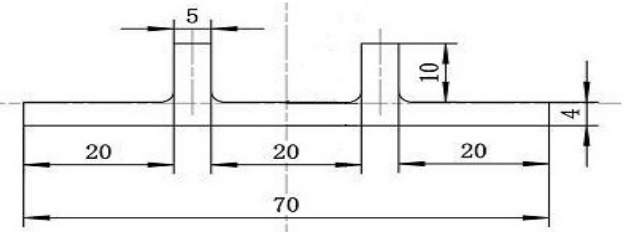

(b)

Fig. 1 The dimension of section profile (a) and die orifice (b)

The cross section area of the excluding profile is $380 \mathrm{~mm}^{2}$, the inner diameter of the extrusion container is $95 \mathrm{~mm}$ and its cross section area is $7084.625 \mathrm{mmm}^{2}$, so the extrusion ratio is 18.64375 .

The cross section shape and size of the profile are one of the important factors of the rational configuration of die holes. The section of the profile in the paper is opposed to a coordinate axis with little difference in thickness, so the section center of the profile should be located on one of the coordinate axis. Then, the center of mass of the profile is calculated by Pappus' theorem according to the die orifice dimension and the cross section of the profile is put in a two-dimensional coordinate system [5].

The cross section of the profile rotates around $\mathrm{X}$ axis to form a cylinder. The area of the cross section is calculated to be $380 \mathrm{~mm}^{2}$. The volume of the cylinder is $9168.8 \mathrm{~mm}^{3}$. The intercept of the center of mass on $\mathrm{Y}$ axis is 3.8; set to $\mathrm{y}$, so the distance gone by the center of mass for one rotation is lower die is shown in Fig. 2.

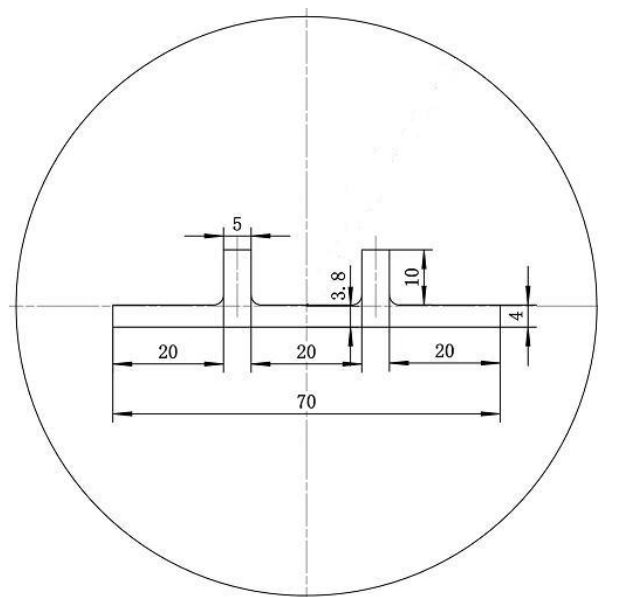

Fig. 2 The layout of single hole and die dimension

\section{Optimization Design of Large-section Profile Die}

The results of flat die are shown in Fig. 3. For large-section profile, the first is to adopt the flat die extrusion at equivalent calibrating strap (Fig. 3 a). The calibrating straps are all $5 \mathrm{~mm}$ and the round at the die orifice is $1 \mathrm{~mm}$. It can be seen that after extrusion, the profile has serious warpage phenomenon (Fig. $3 \mathrm{~b}$ ). This is because the speed at the exit of the profile is not uniform and the velocity of 
T-shaped steel is slower than that of the metal at the center of the container (Fig. $3 \mathrm{c}$ ). In this way, the T-shaped steel at a slower speed is subjected to the compressive stress and the wallboard at a high speed is subjected to the tensile stress, leading to the warping of the profile head.

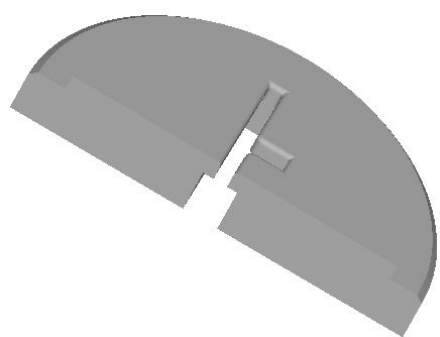

(a)

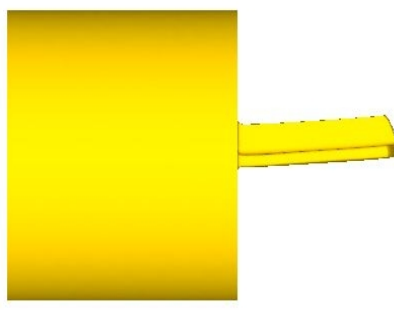

(b)

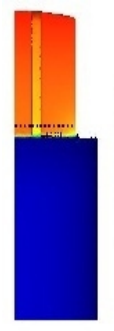

Fig. 3 The deformation procedure using flat die

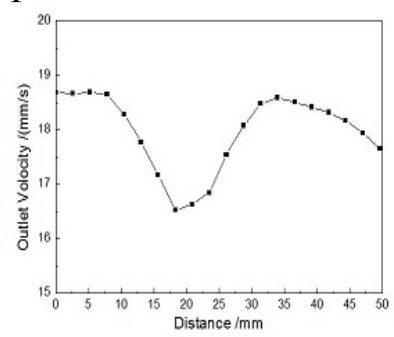

(c)

(a) $3 \mathrm{~d}$ graphics of flat die, (b) extrusion profile, (c) status of extrusion profile export

Adopting the flow guide die can improve the geometrical similarity of billet and profile to easily control the metal flow. Particularly, when the extruding sections of the profile have great different, it can adjust the metal flow velocity to make the profile with thin wall, complex shape and great difficulty easily shaping. Moreover, it can extrude the profile with big circumscribed circle, reduce the product twisting, bending and deformation, improve the stress condition of the die, realize continuous extrusion and thus greatly improve the yield and the die life. The design principle of this kind of die is conductive to the metal pre-distribution and metal flow velocity adjustment, but it has the following disadvantages: the metal needs the secondary deformation and the extrusion stress is higher than that of general flat dies. Fig. 4 is the design dimension of the diversion trench.

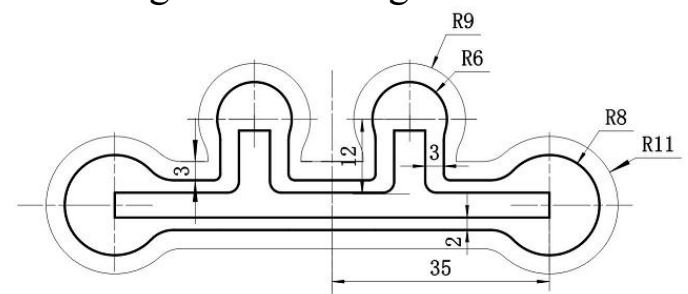

Fig. 4 The hole design of pocket die

To reduce the warping degree of the profile, a diversion trench is added at the place far away from the center of the container to adjust the metal distribution and appropriately make the areas far from the container center get more metals. The results of pocket mold with fixed diameter bearing are shown in Fig. 5. The flow guide die is shown in Fig. 5a, in which the calibrating strap is $5 \mathrm{~mm}$. The extrusion simulation results show that the warping degree of the profile head greatly reduces (Fig. 5b) and the speed difference between T-shaped steel and wallboard reduces (Fig. 5c).

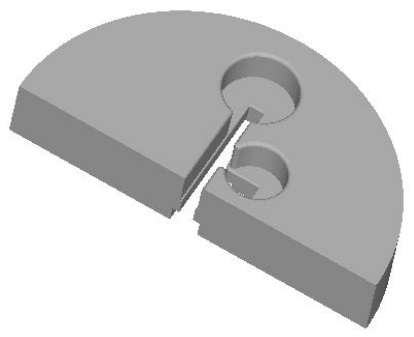

(a)

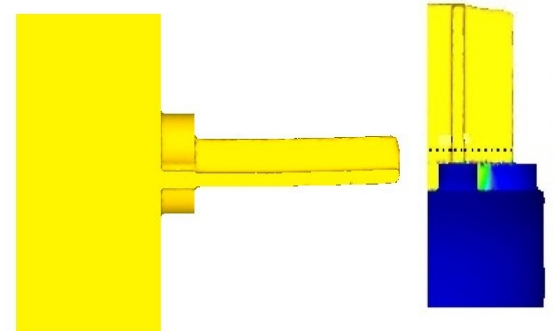

(b)

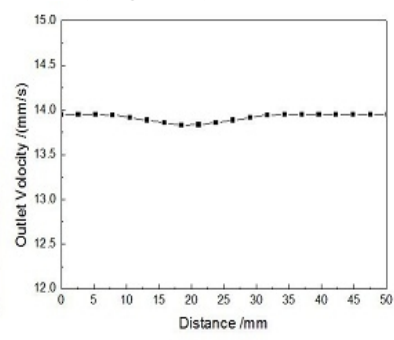

(c)

Fig. 5 The deformation procedure using pocket mold with fixed diameter bearing (a) 3 d graphics of pocket mold with fixed diameter bearing, (b) extrusion profile, (c) status of extrusion profile export

To ensure each point on the profile section flows out of the die hole at the same speed, it needs to reasonably adjust the flow speed of metal. The main method is to reasonably arrange the die holes on the die plane, adopt multi-hole symmetrical arrangement as far as possible and design the calibrating straps of irregular length according to the shape of the profile, the thickness difference between different parts of walls, different specific circumferences and the distance to the center of the container. In general, the thinner the wall at some part of the profile is, the greater the specific 
circumference is, the more complex the shape is and the farther it is from the center of the container, the shorter the calibrating strap at this place is.

The results of pocket mold with gradient diameter bearing are shown in Fig. 6 . The length of the calibrating strap can be determined by the supplementary stress method, as shown in Fig. 6a. It's found after further adjusting the metal flow with the flow guide die with gradational calibrating strap that the speeds at the exit of metal are uniform, the profile head has no warping phenomenon and the whole profile is straight, as shown in Fig. $6 \mathrm{~b}$ and c.

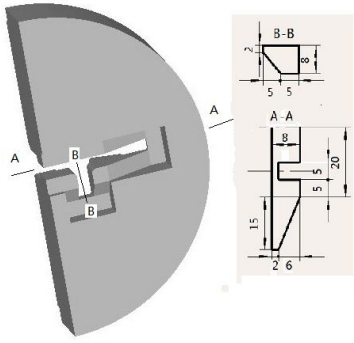

(a)

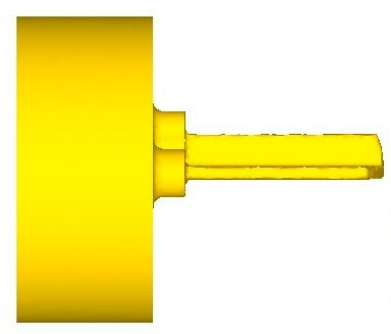

(b)

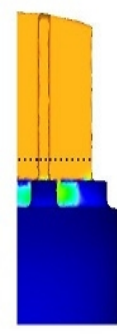

Fig. 6 The deformation procedure using pocket mold with gradient diameter bearing

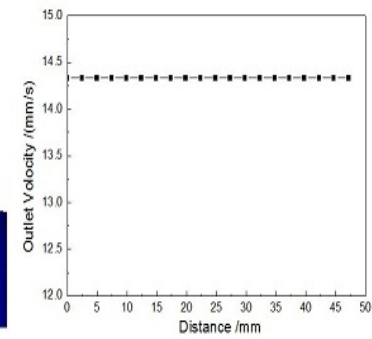

(c) (a) 3 d graphics of pocket mold with gradient diameter bearing, (b) extrusion profile, (c) status of extrusion profile export

After comparison of the loads of dummy blocks under different die designs, the loads are close when the profile is extruded to the stable stage, but the alloy is easier to reach the desired shape through the secondary deformation after adding the diversion trench, as shown in Fig. 7. This is because through the pre-deformation of the diversion trench, the alloy has some dynamic recrystallization, which enhances its plastic deformation ability. Thus, to obtain high-quality profile, the flow guide die with gradational calibrating strap should be adopted to make the numerical simulation on the large-section profile.

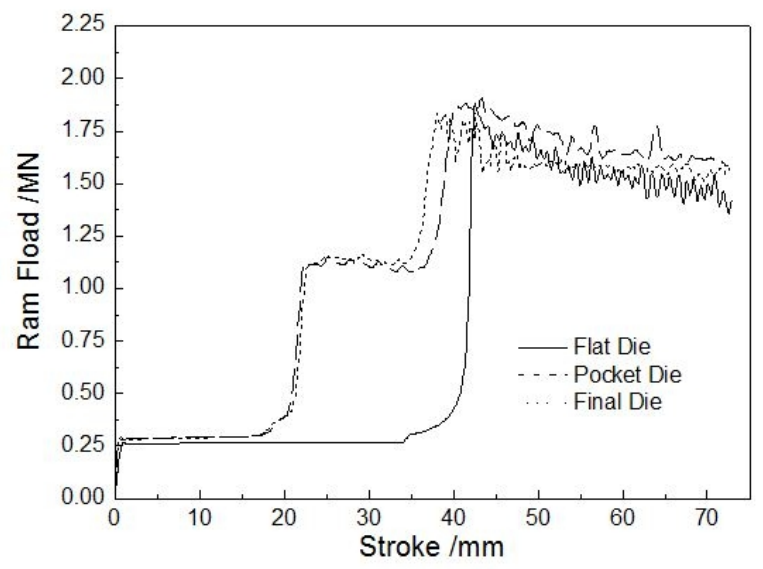

Fig. 7 The ram load of different molds

\section{Summary}

1) It designs the machining size of profile die holes and makes clear the die hoe arrangement.

2) It compares flat die extrusion with equivalent calibrating strap, pocket mold with fixed diameter bearing and pocket mold with gradient diameter bearing and finds that the loads are close when the profile is extruded to the stable stage, but it's easier to reach the desired shape through the secondary deformation after adding the diversion trench.

\section{Acknowledgements}

The authors gratefully acknowledge financial support from NSFC no. 51204020, National Basic Research Program of China no. 2013CB632202 and no. 2013CB632205. 


\section{References}

[1] X.S. Xia, Q. Chen, K. Zhang, Z.D. Zhao, M.L. Ma, X.G. Li, Y.J. Li, Hot deformation behavior and processing map of coarse-grained Mg-Gd-Y-Nd-Zr alloy, Mater. Sci. Eng. A. 587 (2013) 283-290.

[2] N. Srinivasan, Y.V.R.K. Prasad, P. Rama. Rao, Hot deformation behavior of Mg-3Al alloy-A study using processing map, Mater. Sci. Eng. A. 476 (2008) 146-156.

[3] X.H. Wu, G.Q. Zhao, Y.G. Luan, Numerical simulation and die structure optimization of an aluminum rectangular hollow pipe extrusion process, Mater. Sci. Eng. A. (435-436) (2006) 266-274.

[4] L.H. Wang, X.J. Xu, The mathematical basis of the finite element method, Science Press, Beijing, 2005.

[5] G. Fang, J. Zhou, J. Duszczyk. Extrusion of 7075 aluminum alloy through double-pocket dies to manufacture a complex profile, J MATER PROCESSTECH, 209 (2009) 1-10. 